\title{
Acoustic emission entropy: an innovative approach for structural health monitoring of fracture-critical metallic components subjected to fatigue loading
}

\author{
Danilo D'Angela ${ }^{1 *}$ and Marianna Ercolino ${ }^{2}$ \\ ${ }^{1}$ University of Naples Federico II, Department of Structures for Engineering and Architecture, Via Claudio 21, \\ 80125 Naples, Italy. \\ ${ }^{2}$ University of Greenwich, School of Engineering, Central Avenue ME4 4TB, Chatham, United Kingdom.
}

*Corresponding author: danilo.dangela@unina.it

\begin{abstract}
The paper presents an innovative approach for structural health monitoring of metallic components under fatigue crack phenomena. The methodology is based on the evaluation of the information entropy of the AE data. AE testing of fatigue crack growth (FCG) is performed on metallic components is performed within an extremely noisy testing environment. Basic AE data analysis is demonstrated to be inefficient with regard to the specific testing conditions. AE entropy is proven to be a reliable damage-sensitive feature for real-time assessment despite both significant noise disturbance and complexity/randomness of the acoustic phenomena. This was also confirmed for (time-)discontinuous monitoring processes over random-based data detections. An innovative monitoring protocol is finally developed according to the experimental evidence also considering the recommendations of the current monitoring. The protocol is found to be promising for structural health monitoring of metallic fracture-critical components of structures under fatigue.
\end{abstract}

KEYWORDS: structural health monitoring; acoustic emission, information entropy; fatigue crack; metallic components

\section{INTRODUCTION}

The risk of economic and human losses related to infrastructure failure significantly increased in the last decades. This was essentially due to (a) the expansion of the infrastructure network, (b) the increased performance demands, and (c) the degradation of existing systems. ${ }^{1}$ Infrastructural systems such as bridges, pipelines, and power/communication facilities are among the most critical structures because of their high vulnerability. ${ }^{2,3}$ The mechanical degradation of one or few components of such structures is likely to cause a global performance deficiency, with a resulting catastrophic reduction in structural safety. ${ }^{4}$ 


\section{Nomenclature}

\begin{tabular}{|c|c|}
\hline $\mathrm{a}$ & experimental coefficient (b-value analysis) \\
\hline A & AE amplitude \\
\hline B & sample thickness \\
\hline $\mathrm{b}_{\mathrm{AE}}$ & b-value \\
\hline $\mathrm{f}$ & cyclic frequency \\
\hline $\mathrm{H}$ & sample height \\
\hline $\mathrm{j}$ & number of occurred potential crack onset alarm \\
\hline $\mathrm{k}$ & number of occurred definite crack onset alarm \\
\hline 1 & number of occurred potential failure alarm \\
\hline $\mathrm{m}$ & number of occurred definite failure alarm \\
\hline $\mathrm{k} \Delta 0$ & crack onset test coefficient \\
\hline $\mathrm{k}_{\Delta \mathrm{F}}$ & failure test coefficient \\
\hline $\mathrm{LT}_{\mathrm{T}}$ & component structural lifetime \\
\hline $\mathrm{n}$ & number of AE counts \\
\hline $\mathrm{n}_{\mathrm{i}}$ & number of $\mathrm{AE}$ counts related to $\mathrm{i}^{\text {th }}$ time step \\
\hline $\mathrm{N}$ & number of AE hits \\
\hline $\mathrm{N}_{\text {DW }}$ & number of detection windows over the whole monitoring process \\
\hline $\mathrm{N}_{\mathrm{L}}$ & sample notch length \\
\hline$\overline{\mathrm{N}}$ & number of AE hits having amplitude not smaller than a certain threshold \\
\hline $\mathbf{p}_{\mathbf{i}}$ & probability mass distribution vector \\
\hline $\mathrm{P}$ & cyclic peak load \\
\hline $\mathrm{R}$ & minimum to maximum stress ratio \\
\hline $\mathrm{t}$ & time \\
\hline $\mathrm{t}_{0}$ & crack onset time \\
\hline $\mathrm{t}_{\mathrm{f}}$ & failure time \\
\hline $\mathrm{T}$ & $\begin{array}{l}\text { time interval from the end of the current detection window to the beginning of } \\
\text { the following }\end{array}$ \\
\hline $\mathrm{T}_{\mathrm{DW}}$ & duration of each detection window \\
\hline $\mathrm{T}_{\mathrm{T}}$ & total $\mathrm{AE}$ detection time \\
\hline $\mathrm{T}_{1}, \mathrm{~T}_{2}, \mathrm{~T}_{3}$ & specific time interval values associated with different response to the crack \\
\hline $\mathrm{T}_{4}, \mathrm{~T}_{5}$ & onset and failure tests \\
\hline$\underline{T}$ & inspection time interval vector \\
\hline $\bar{S}_{\mathrm{E}}$ & Shannon entropy \\
\hline $\mathrm{SE}_{\mathrm{E}, \mathrm{r}}$ & Kullback-Leibler or relative entropy \\
\hline $\mathrm{W}$ & sample width \\
\hline$\underline{\Delta}$ & onset/failure test response vector \\
\hline$\underline{\overline{\Delta S}}$ & entropy variation value vector \\
\hline$\overline{\Delta \Sigma} \mathrm{S}_{\mathrm{E}, \mathrm{i}}$ & variation of the cumulative entropy over the whole $\mathrm{i}^{\text {th }}$ detection window \\
\hline$\Delta \Sigma \mathrm{S}_{\mathrm{E}, 345}$ & $\begin{array}{l}\text { average of the values related to the } 3^{\text {rd }}, 4^{\text {th }} \text {, and } 5^{\text {th }} \text { detection window variations } \\
\text { evaluated over the entire monitoring process }\end{array}$ \\
\hline$\Sigma \mathrm{n}$ & cumulative number of AE counts \\
\hline$\sum \mathrm{n}_{\mathrm{i}}$ & cumulative number of AE counts related to ith time step \\
\hline$\Sigma \mathrm{T}_{\mathrm{F}}$ & failure alarm time \\
\hline
\end{tabular}

The issue is particularly sensitive for metallic fracture-critical members. ${ }^{5,6}$ As an example, metallic bridge structures were the typology that suffered the largest number of collapses all over the world in the last few decades. ${ }^{7,8}$ The combination of fatigue and fracture caused most 
of those failures and typically represents the potential failure mode also in the case of existing bridges. ${ }^{9}$ Fatigue and fracture combination is a common cause of severe damage and degradation in pipelines as well. ${ }^{6}$ The early crack detection in infrastructures plays a crucial role in the mitigation of the risk associated with damage and collapse. In fact, fatigue crack growth processes can be quite slow, but the incipient critical state is often reached with reduced deformations ${ }^{10}$, and the failure can occur even without an evident (large-scale) plastic response $^{11}$.

Structural health monitoring ${ }^{12}$ (SHM) is aimed to assess the health state of infrastructures. ${ }^{13,14}$ In the past decades, a major part of the monitoring activities was based on in-situ inspections and destructive testing. This approach was usually uneconomical as well as possibly unreliable. ${ }^{9,10}$ Several non-destructive techniques (NDTs) were recently developed to reduce the invasiveness/costs of the monitoring by keeping damage diagnosis and health control effective. ${ }^{15,16}$ Acoustic emission (AE) testing is an innovative passive NDT often used to assess bridges and other (infra)structures. ${ }^{16-19}$ As a passive technique, AE testing is performed by recording signals over the structures during their lifetime. In particular, genuine acoustic waves are typically generated in solids due to damage and degradation occurrence (e.g., cracking initiation and propagation ${ }^{10}$ ). The AEs propagate within the bodies interacting with the structural discontinuities. The detection, the processing, and the analysis of the AE activity allow assessing the state-of-health of the monitored component/system. ${ }^{1,19-22}$ A schematic representation of the testing application is shown in Fig. 1.a. AE testing can be performed by using either the parameter-based (or classical) or the signal-based (or quantitative) approach. ${ }^{18}$ The two approaches are typically used in different applications. According to the parameterbased approach, the AE activity can be described by a set of parameters of the waveforms, namely the AE features. The main AE features are shown in Fig. 1.b.

The signal-based approach builds on a more comprehensive analysis of the waveforms, as well as on more complex post-processing phases. Therefore, this approach is not easily applicable to real-time monitoring. This is often used for either (post-event) localisation of the damage source or frequency analysis of the waveforms. The parameter-based approach is effectively considered for real-time monitoring of structures because of (a) the reduced amount of data to be stored, (b) the automated analysis implementation, (c) the proven accuracy for damage assessment, and (d) the availability of several data analysis/processing techniques. 


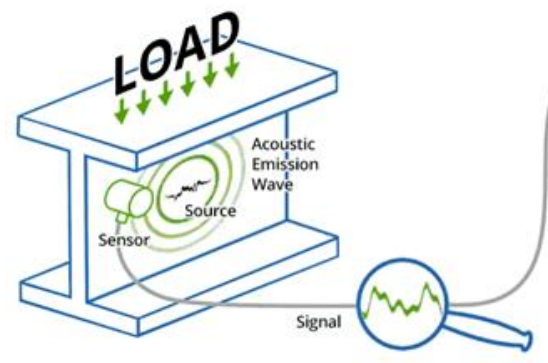

(a)

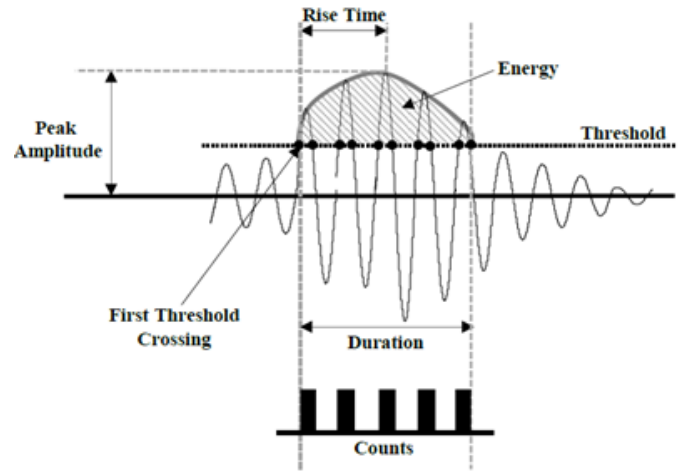

(b)

Fig. 1. AE testing: (a) technique application scheme (by Mistras LTD) and (b) main AE features. $^{23}$

AE testing was widely applied in the last few decades, and several studies demonstrated the reliability and robustness of the technique over a wide range of laboratory and field applications. Some in situ applications proved that the analysis of the AE activity might overcome the noise disturbance due to environmental or traffic conditions. The pioneering study by Shigeishi et al. ${ }^{24}$ assessed AE testing for masonry and reinforced concrete bridge monitoring, proving that the AE analysis potentially allows detecting crack propagation as well as localising crack tips. Carpinteri et al. ${ }^{21}$ correlated the AE activity to the extending crack size of macrocracks propagating in a multistorey reinforced concrete building. AE testing was often applied to assess concrete, rock, and masonry, but some studies proved that the technique could also be reliable with regard to metallic structures subjected to fracture phenomena. Maslouhi ${ }^{19}$ correlated direct $\mathrm{AE}$ features and frequency-based wave parameters to fatigue crack initiation/propagation occurring in aluminium alloy structures. Pascoe et al. ${ }^{25}$ supplied insights into the acoustic activity generated by crack propagation over a single fatigue cycle by testing double-cantilever beam specimens made of aluminium alloy. They stressed the potential of AE testing for a more robust assessment of crack propagation phenomena as well as the need to enhance the technique. Wisner et al. ${ }^{26}$ recently assessed the progressive failure process in a precipitate hardened aluminium alloy by performing AE testing combined with scanning electron microscope and X-ray microtomography analyses. They found clear correlations between the microdamage evolution and the AE data.

The ideal application of AE testing is the (time-)continuous monitoring of the structure from the construction (or the beginning of the service/functioning) along with the lifetime. This condition is not often met in field monitoring because of (a) the high costs of the monitoring and the needed expertise, (b) the sensitivity of the testing equipment to environmental conditions and ageing, and (c) a large number of structures to be potentially monitored. Most 
experimental studies focussed on (time-)continuous or almost (time-)continuous AE data, simulating a lifetime-long AE testing. Both AE testing application and data analysis should still be improved for more efficient monitoring. ${ }^{27,28}$ Some processing techniques were recently developed to reduce the equipment/environmental noise typically affecting the $\mathrm{AE}$ data. However, the signal disturbance strongly depends on the specific application and monitoring conditions, and field monitoring is often needed to identify the noise patterns. ${ }^{29}$ Structural damage potentially has distinctive AE features that should be experimentally identified. ${ }^{29}$ This becomes quite difficult in the case of fatigue fracture assessment ${ }^{30,31}$, with particular reference to (a) a real structure/infrastructure under service/functioning conditions (i.e., extremely noisy environment) ${ }^{13,27,32}$ and (b) (time-)discontinuous or periodic data detection. Hence, novel approaches and methods are needed to address this critical issue. ${ }^{15,33}$

\section{DAMAGE, ACOUSTIC EMISSION, AND ENTROPY}

The damage/degradation within solids is a source of microstructural disorder and energy dissipation. The AE activity reflects such disorder along with the evolution of the complexity of an engineering system. ${ }^{34}$ The analysis of the systemic disorder has been recently proven to be a reliable method for damage detection of structures, with particular regard to the evaluation of the systemic entropy. ${ }^{35-37}$ Entropy is an extensive property of a thermodynamic system. In information theory, entropy describes the amount of information contained within a system, e.g., the potential number of microstates that the system can assume. The computation of the information entropy is used in statistical mechanics as a measure of the disorder of an engineering system. Shannon entropy ${ }^{38}$ is among the first developed formulations of the information entropy. This defines the measure of uncertainty contained within a random variable, which can be quantified by the amount of information stored by the variable. The information entropy of $\mathrm{AE}$ data is often defined as AE entropy or acoustic entropy. The evaluation of the acoustic entropy does not directly involve the thermodynamic entropy; however, the two entropy forms are correlated, and their applied formulations are substantially equivalent. Furthermore, acoustic entropy can be considered as a portion of the total thermodynamic entropy. ${ }^{34}$

AE entropy can be a reliable feature for the assessment of structural damage. Unnthorsson et al. ${ }^{39}$ evaluated the Shannon entropy of acoustic data in cyclic tests of carbon fibre reinforced polymers. AE entropy identified the cyclic damage more efficiently than the traditional AE features. Kahirdeh and Khonsari ${ }^{31}$ found statistical correlations between cyclic material degradation, temperature, and acoustic energy dissipation. The same Authors ${ }^{40}$ assessed the 
acoustic entropy generated by cyclic bending tests on metallic and composite elements. A clear quantitative correlation was found between the entropy and the evolution of fatigue degradation. Kahirdeh et al. ${ }^{34}$ focussed on the use of AE entropy for health monitoring purposes. The best damage-correlated probability functions for the AE entropy evaluation were found by maximising the cumulative AE entropy related to cyclic tensile testing of aluminium samples. A measure of the relative acoustic entropy was also assessed considering the Kullback-Leibler divergence ${ }^{41}$, assuming that each AE event provides prior information regarding the consequent event. The relative entropy exhibited a clearer correlation with the fatigue damage than the Shannon one. Sauerbrunn et al. ${ }^{20}$ computed the AE entropy for the signal voltage of single $\mathrm{AE}$ waveforms similarly to Kahirdeh et al. ${ }^{33,34}$. The AE entropy evaluation resulted better correlated to the fatigue damage than the traditional AE features with reference to both small and large cracks. Chai et al. ${ }^{32}$ also computed the Shannon AE entropy considering the voltage signal of the single AE events. They characterised the different damage stages of fracture for both (a) fracture crack growth tests and (b) three-point bending tests. Yu and Modarres $^{37}$ recently confirmed that the Shannon entropy of the AE activity could be associated with the fatigue damage better than the conventional AE features such as the counts and the energy. Some very recent studies enhanced the assessment of the AE entropy and provided criteria potentially applicable to health monitoring of metallic structures. ${ }^{42,43}$

Even though AE Entropy is potentially reliable for damage assessment of structures, the testing conditions for the field monitoring of metallic fracture-critical structures have still to be effectively addressed. Moreover, the past experimental studies were performed in a noisecontrolled environment where the sensors were directly located on the damaging component without taking into account the chaotic nature of the AE phenomena. Damage criteria based on periodic monitoring should still be provided in the framework of standardised protocols; for example, some parameters of the monitoring process (e.g., time for the following inspection) with $\mathrm{AE}$ should take into account the current state of the monitored components.

The paper presents a preliminary application of a monitoring protocol of fatigue damage in metallic components within a noisy testing environment. AE testing of fatigue crack growth (FCG) in metallic compact tension (CT) specimens was performed. The preliminary results of the present study were presented in a conference paper ${ }^{44}$. AE entropy is evaluated by using both Shannon ${ }^{38}$ and Kullback-Leibler ${ }^{41}$ equations. Experimental criteria based on AE entropy are established for different damage states: crack initiation, propagation, and failure. The efficiency of the criteria is assessed by simulating a real application with time-discontinuous monitoring of AE data (e.g., periodic monitoring). 


\section{EXPERIMENTAL TESTING AND DATA ANALYSIS}

Constant-amplitude cyclic tests were performed on CT specimens. The plate materials were 7075-T6 aluminium alloy (yield strength (0.2\%) equal to $505 \mathrm{MPa}$ EN 485 / EN 10204 3.1) and S355 steel (yield strength equal to $355 \mathrm{MPa}, E N$ 10025-2). AE testing was performed according to the parameter-based approach along with the mechanical tests. The cyclic tests were carried out using a servo-hydraulic actuator controlled by a modular digital system. The AEs were recorded by a pre-amplified ultra-low noise sensor with a resonance frequency equal to $300 \mathrm{kHz}$ (PK30I sensor). The sensor was coupled/bonded to the fixed end of the fatigue machine by a thin layer of silicon glue (Loctite 595), with a safety tie by adhesive tape. This peculiar location of the sensor was aimed to detect AE data affected by significant noise due to the fatigue testing equipment. The sensor location simulated an application in which the genuine data (i.e., damage-induced) are overlapped by significant noise, which does not have known patterns. The data were stored and processed by a single-channel USB AE Node system, using $A E$ win $^{\mathrm{TM}}$ as a software. Both the AE equipment (including the software) and the sensor were produced by Mistras LTD. The experimental set-up and the sensor location are shown in Fig. 2.a. Both the sensitivity and the efficiency of the sensor coupling were checked by pencil lead break test ${ }^{45}$ prior to the main experiments. Two preliminary fatigue tests on aluminium 7075-T6 samples were performed to select the main AE testing parameters. According to those tests, the AE amplitude threshold was set equal to $45 \mathrm{~dB}$. The peak definition time, the hit definition time, and the hit lockout time were set equal to 200,800 , and $1000 \mu$ s, respectively. The main tests included four CT specimens (Table 1 and Fig. s 2.b and 2.c).

(a)

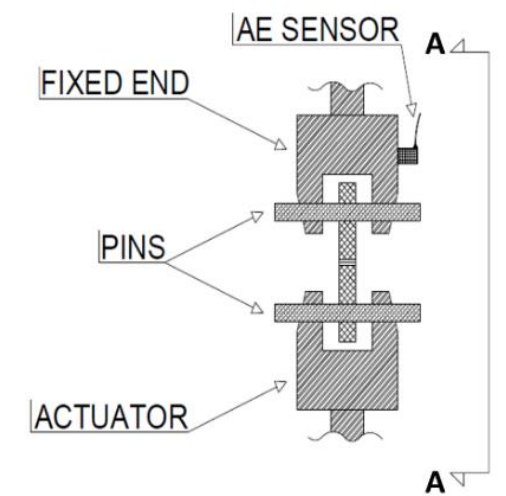

Section $B-B$

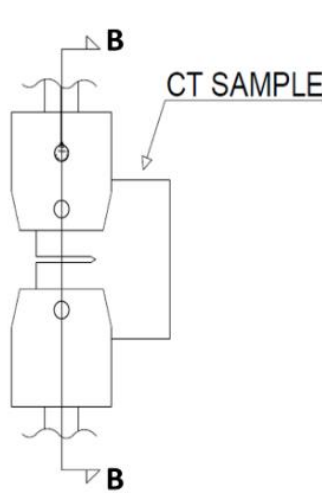

Section A - A

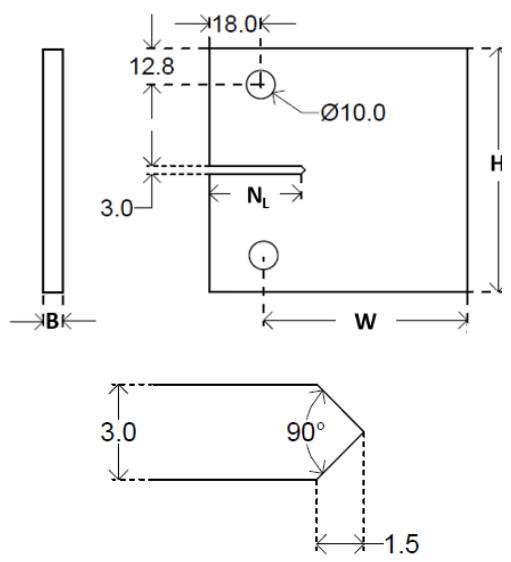

(b)

(c)

Fig. 2. (a) Testing set-up and sensor location, (b) sample geometry, and (c) sample notch detail. The dimensions are in $\mathrm{mm}$. 
Two aluminium samples with different geometry were tested under different cyclic peak loads $P$ (tests $A_{1}$ and $A_{2}$ ). Two steel samples with the same geometry were tested under different cyclic peak loads $P$ (tests $S_{1}$ and $S_{2}$ ). All tests were performed considering the same $R$ ratio (i.e., minimum to maximum stress ratio) and cyclic frequency $f$. Fig. 2.b shows the geometry of the samples, and Table 1 reports the geometrical parameters $\left(W, H, N_{L}\right.$, and $\left.B\right)$ and the testing features $(P, R$, and $f)$ related to the tests.

Table 1. Sample geometry and loading program.

\begin{tabular}{lllllllll}
\hline test & material & $\boldsymbol{W}$ & $\boldsymbol{H}$ & $\boldsymbol{N}_{\boldsymbol{L}}$ & $\boldsymbol{B}$ & $\boldsymbol{P}$ & $\boldsymbol{R}$ & $\boldsymbol{f}$ \\
\cline { 3 - 8 } ID & & {$[\mathrm{mm}]$} & {$[\mathrm{mm}]$} & {$[\mathrm{mm}]$} & {$[\mathrm{mm}]$} & {$[\mathrm{kN}]$} & {$[-]$} & {$[\mathrm{Hz}]$} \\
\hline$A_{1}$ & 7075-T6 aluminium alloy & 71 & 86 & 32 & 4 & 2.5 & 0.05 & 10 \\
\hline$A_{2}$ & 7075-T6 aluminium alloy & 63 & 77 & 29 & 10 & 5 & 0.05 & 10 \\
\hline$S_{1}$ & S355 steel & 63 & 77 & 29 & 10 & 5 & 0.05 & 10 \\
\hline$S_{2}$ & S355 steel & 63 & 77 & 29 & 10 & 6 & 0.05 & 10 \\
\hline
\end{tabular}

The crack length was measured during the tests by means of a calliper, highlighting the cracks by dye penetrant testing ${ }^{46}$. High-resolution images of the fracture zone were regularly taken to evaluate the crack propagation by image post-processing as well. The crack length was highlighted by the crack developer, and it was clearly visible. The crack onset was conventionally defined by the first time in which the crack length equalled or exceeded the given accuracy of the measuring tools $(\sim 1.0 \mathrm{~mm})$. The failure was conventionally defined as the complete fracture of the sample.

The AE data were initially filtered according to the modified Swansong II technique ${ }^{23,47}$ in order to reduce the noise disturbance. This filtering criterion builds on the evidence that (a) genuine long-duration hits usually have higher amplitude and (b) genuine short-duration hits usually have lower amplitude. ${ }^{48}$ The assumed amplitude and duration filtering limits are shown in Table 2; they were derived from past experimental studies ${ }^{47}$ on similar components.

Table 2. Amplitude and duration limits for the application of the modified Swansong II filtering.

\begin{tabular}{llllllllll}
\hline amplitude & {$[\mathrm{dB}]$} & $<52.5$ & $<55.0$ & $<57.5$ & $<60.0$ & $<62.5$ & $<65.0$ & $<70.0$ & $<75.0$ \\
duration & {$[\mathrm{ms}]$} & $>0.5$ & $>1.5$ & $>2.5$ & $>3.5$ & $>4.5$ & $>5.5$ & $>6.5$ & $>7.0$ \\
\hline
\end{tabular}

The traditional analysis of the AE was performed by means of both historical plots and correlation analysis. ${ }^{45,49}$ The $b$-value analysis was performed as a refined correlation analysis method. ${ }^{35,50}$ This is based on the empirical magnitude-frequency relationship proposed by Gutenberg and Richter ${ }^{51}$ in earthquake seismology; $b$-value (or $b_{A E}$ ) is the (negative) gradient 
of the log-linear AE magnitude-frequency distribution of the AE hits. Magnitude is conventionally assumed to be equal to amplitude in $\mathrm{dB}(A)$ divided by 20 . The $b$-value correlation is defined by

$$
\log _{10} \bar{N}=\mathrm{a}-\mathrm{b}_{\mathrm{AE}} \frac{\mathrm{A}}{20},
$$

where $\bar{N}$ is the number of hits having the amplitude not smaller than a certain value $(A$ in Equation 1), and $a$ is a coefficient to be experimentally evaluated by data fitting. Analysis of $b$-value was widely proven to be reliable in rocks, concrete, and reinforced concrete elements. ${ }^{35,52}$ Few recent applications also showed that $b$-value could identify the damage evolution in metallic components under fracture. ${ }^{53,54}$

Shannon entropy $\left(S_{E}\right)$ of the AE data was calculated at each time step (i) according to the following equation ${ }^{38,40}$ :

$$
\mathrm{S}_{\mathrm{E}}=-\sum_{\mathrm{i}=1}^{\mathrm{n}} \mathrm{p}_{\mathrm{i}} \log _{2}\left(\mathrm{p}_{\mathrm{i}}\right)
$$

in particular, $\boldsymbol{p}_{\boldsymbol{i}}$ is the probability mass distribution vector:

$$
\mathbf{p}_{\mathbf{i}}=\left\{\frac{\mathrm{n}_{1}}{\Sigma \mathrm{n}_{\mathrm{i}}} ; \frac{\mathrm{n}_{2}}{\Sigma \mathrm{n}_{\mathrm{i}}} ; \ldots ; \frac{\mathrm{n}_{\mathrm{i}}}{\Sigma \mathrm{n}_{\mathrm{i}}}\right\}
$$

where, $n_{j}$ is the measured number of counts and $\Sigma n_{i}$ is the cumulative counts related to the $i^{\text {th }}$ time instant in which acoustic activity is detected. This calculation assumes that each individual AE signal does not affect the previous ones. ${ }^{34}$ The Kullback-Leibler formulation ${ }^{41}$ was used to estimate a relative measure of the AE entropy, i.e., $S_{E, r}{ }^{34}$ :

$$
\mathrm{S}_{\mathrm{E}, \mathrm{r}}=\mathrm{S}_{\mathrm{E}}\left(\mathrm{p}_{\mathrm{i}} \mid \mathrm{p}_{\mathrm{i}-1}\right)=-\sum_{\mathrm{i}=1}^{\mathrm{n}} \mathrm{p}_{\mathrm{i}} \log _{2}\left(\frac{\mathrm{p}_{\mathrm{i}}}{\mathrm{p}_{\mathrm{i}-1}}\right)
$$

The cumulative values of both Shannon entropy and relative entropy are defined as $\Sigma S_{E}$ and $\Sigma S_{E, r}$, respectively. $S_{E}$ and $S_{E, r}$ were computed using Equations 2 and 4 respectively, for each testing time step defining the (time-) continuous entropy, which is consistent with a monitoring process continuously performed over the lifetime of the structure over cyclic loading. However, health monitoring is often based on (time-)discontinuous or periodic testing processes as it was previously discussed. Therefore, a periodic AE entropy evaluation was implemented according to the procedure that is described in the relevant section (par. 3.3) for the sake of clarity. 


\section{RESULTS AND DISCUSSION}

\subsection{Basic acoustic emission analysis}

The historical plots of counts $(n)$ and cumulative counts $\left(\sum n\right)$ are shown in Fig. 3 for (a) $A_{l}$, (b) $A_{2}$, (c) $S_{1}$, and (d) $S_{2}$ cases. The counts show a different trend along with the time for the tested samples. Larger sample thickness (peak load) generates more AE activity, as it is evidenced by comparing $A_{2}$ with $A_{1}\left(\mathrm{~S}_{2}\right.$ and $\left.S_{1}\right)$. The overall $\mathrm{AE}$ activity is more significant in aluminium samples, and it might be justified by the lower fracture toughness of this material. The counts are correlated to the crack onset in the case of aluminium samples, especially for the $A_{l}$ case: significant AE activity is detected after the crack onset (Fig. s 3.a and 3.b). Such a correlation cannot be found in the steel samples: significant $\mathrm{AE}$ activity is detected before the crack onset (Fig. 3.c and Fig. 3.d) without a clear relationship between the counts and the incipient fracture damage. On the contrary, the fracture failure is associated with sudden AE activity in both materials. The sudden change in the AE activity just prior to the failure (less relevant in the $A_{1}$ case) does not allow to predict the failure itself; therefore, this correlation is not potentially applicable for structural monitoring. In many cases, the occurrence of significant AE activity is not correlated to damage and degradation (e.g., Fig. 3.b at about 1000 s, and Fig. 3.d at about 200 s). Such activity caused false alarms, which are typically observed in AE testing. ${ }^{23}$ Similarly, other historical plots and (direct) AE feature correlation plots (e.g., duration versus amplitude) do not show univocal and reliable damage correlations; such results are not reported within the paper for the sake of brevity.

Fig. 4 shows the results of the $b$-value analysis for the performed experimental tests. The analysis time ranges were selected considering the identified sub-stages of the AE data time histories, i.e., according to the similarities among the time-binned data (Table 3). A good damage correlation is found for aluminium sample cases (Fig. s 4.a and 4.b, and Table 3), where $b$-value decreases as the damage increases, and it suddenly reaches low values equal to about 0.6 just prior to the failure (typical value for fracture failure ${ }^{35}$ ). Conversely, this method is not efficient in the case of steel samples (Fig. s 4.c and 4.d), where such a pattern is not observed. It is worth noting that in these latter cases, the acoustic activity does not present large amplitude events during the damage evolution as well as prior to the failure. The typology of the tested samples might partially justify the inefficiency of the $b$-value analysis results. The crack/degradation development in metallic plates is usually a quite regular phenomenon, usually occurring according to a (unique) unidimensional path. The $b$-value analysis can be 
very efficient in case of compact composite components under fracture, such as concrete beams, in which stages of micro-cracking and macro-cracking use to occur. ${ }^{35,50}$

(a)

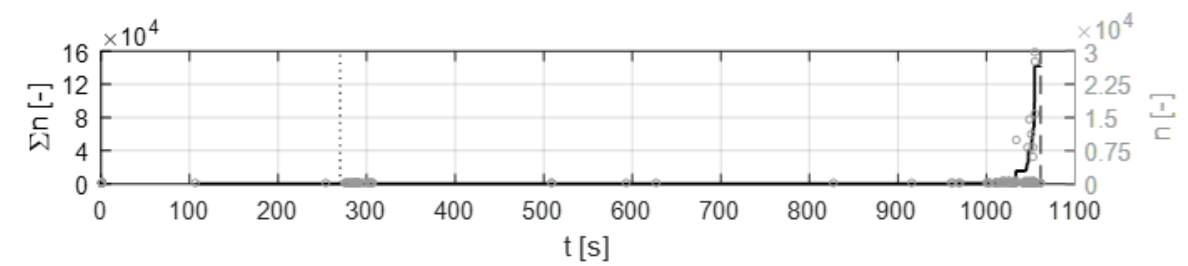

(b)

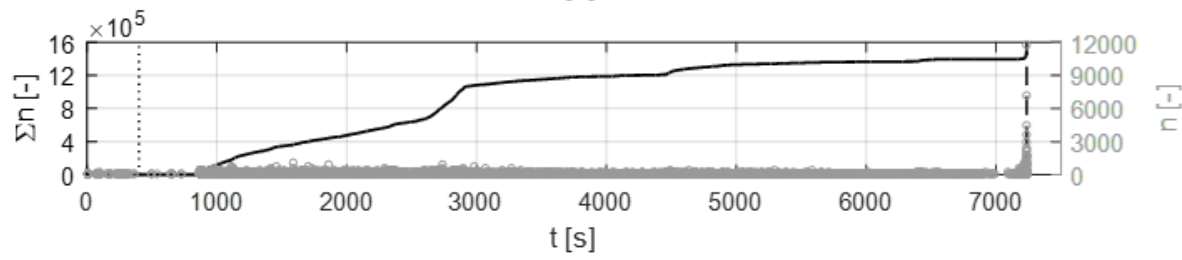

(c)

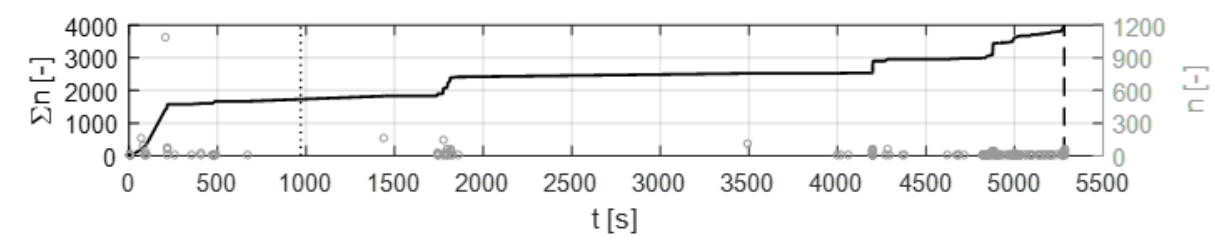

(d)

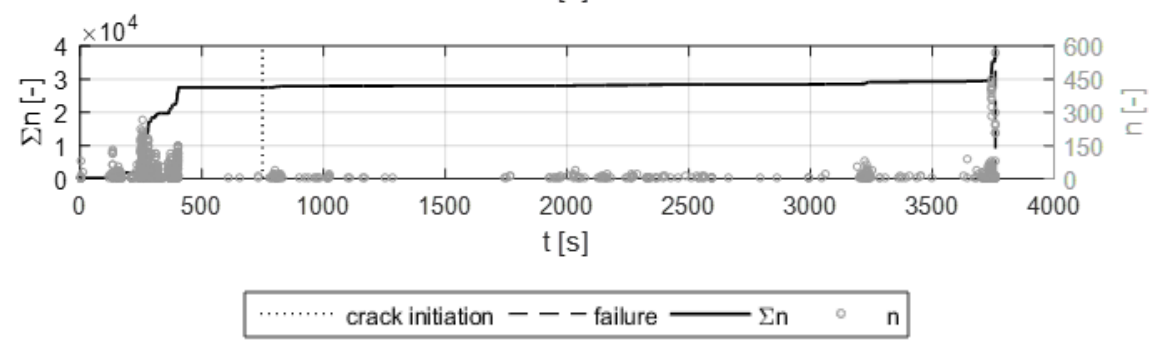

Fig. 3. Historical plots of counts (n) and cumulative counts ( $\Sigma n$ ) for (a) $A_{1}$, (b) $A_{2}$, (c) $S_{1}$, and (d) $S_{2}$. Crack initiation and failure times are also shown.

The results of the basic analysis demonstrate the reliability of the performed tests, i.e., qualitative damage correlations are representative of genuine testing. However, the analysis cannot univocally assess the damage because of both false alarms and missed degradation phases. Such inefficiency is likely to be caused by the noise/disturbance. Indeed, it is worth recalling that the sensor was located on the fatigue machine instead of on the sample; therefore, the influence of the mechanical equipment disturbance on the basic AE activity was expected. The mechanical noise seems to significantly affect the AE data despite the signal filtering actions due to (a) sensor response, (b) amplitude threshold, and (c) modified Swansong II filtering. The results also stress the chaotic nature of the AEs, which is expected to be more significant in the case of field applications. Even though the fracture damage produces acoustic waves that may univocally identify the damage evolution, such genuine AEs are partially/totally affected by the mechanical and environmental noise. Furthermore, the AE data 
interpretation becomes more complex due to the chaotic nature of both fracture damage and acoustic phenomena.
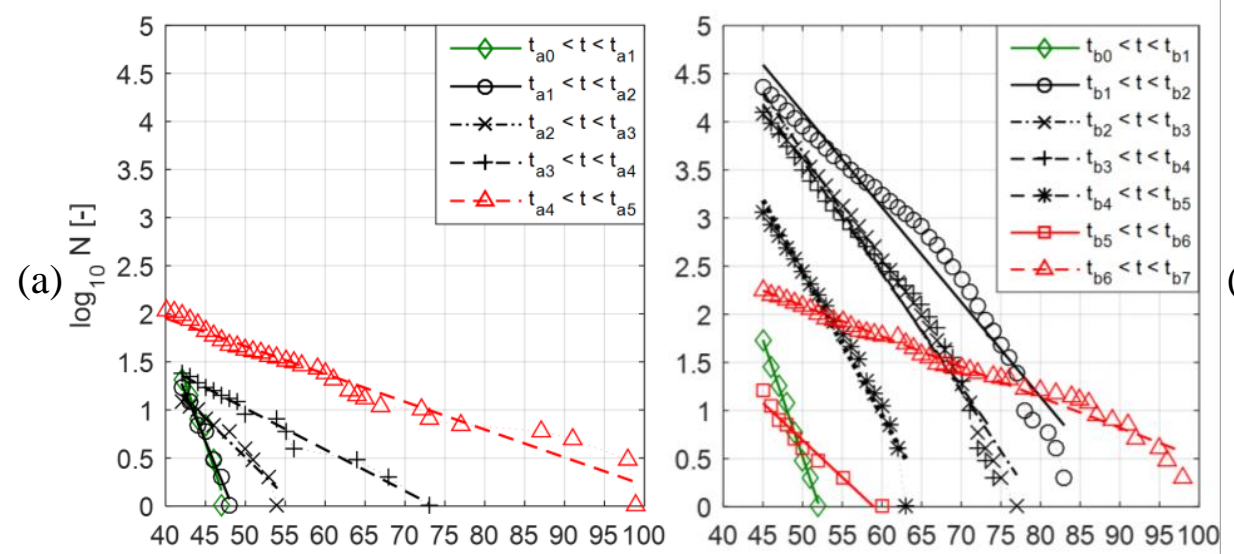

(b)
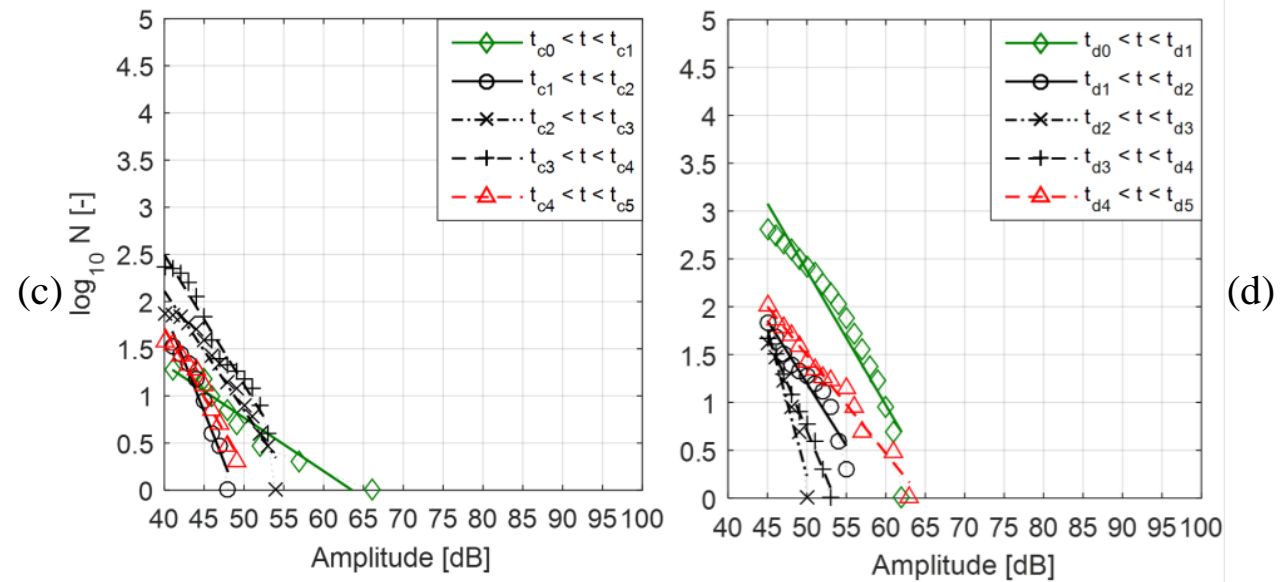

Fig. 4. AE $b$-value analysis results for (a) $A_{1}$, (b) $A_{2}$, (c) $S_{1}$, and (d) $S_{2}$. The time ranges and the $b$-values $\left(b_{A E}\right)$ are reported in Table 3.

Table 3. $b$-value analysis results: time intervals and $b$-values $\left(b_{A E}\right)$.

\begin{tabular}{lllllllll}
\hline $\boldsymbol{i}$ & $\boldsymbol{t}_{\boldsymbol{a}}$ & $\boldsymbol{t}_{\boldsymbol{b}}$ & $\boldsymbol{t}_{\boldsymbol{c}}$ & $\boldsymbol{t}_{\boldsymbol{d}}$ & $\boldsymbol{b}_{\boldsymbol{A E E , \boldsymbol { a }}}$ & $\boldsymbol{b}_{\boldsymbol{A E , \boldsymbol { b }}}$ & $\boldsymbol{b}_{\boldsymbol{A E}, \boldsymbol{c}}$ & $\boldsymbol{b}_{\boldsymbol{A E , \boldsymbol { d }}}$ \\
\hline$[-]$ & {$[\mathrm{s}]$} & {$[\mathrm{s}]$} & {$[\mathrm{s}]$} & {$[\mathrm{s}]$} & {$[-]$} & {$[-]$} & {$[-]$} & {$[-]$} \\
\hline 0 & 106 & 0 & 4 & 4 & 4.96 & 4.87 & 1.13 & 2.81 \\
\hline 1 & 298 & 723 & 474 & 405 & 4.01 & 1.97 & 4.27 & 2.61 \\
\hline 2 & 1002 & 2600 & 1740 & 2024 & 1.62 & 2.49 & 2.52 & 6.13 \\
\hline 3 & 1020 & 2950 & 4670 & 3200 & 0.86 & 2.35 & 2.76 & 3.99 \\
\hline 4 & 1043 & 6400 & 5165 & 3710 & 0.58 & 2.99 & 2.93 & 2.04 \\
\hline 5 & 1061 & 6920 & 5281 & 3760 & - & 1.54 & - & - \\
\hline 6 & - & 7180 & - & - & - & 0.63 & - & - \\
\hline 7 & - & 7235 & - & - & - & - & - & - \\
\hline
\end{tabular}

\subsection{Acoustic entropy}

Cumulative Shannon and relative entropies $\Sigma S_{E}$ and $\Sigma S_{E, r}$ are plotted in Fig. 5.a and 6.b, respectively, for all the performed tests along with time $(t)$ divided by failure time $\left(t_{f}\right)$. The 
experimental crack onset time $t_{0}$ is also shown by vertical lines. Both entropy formulations show a similar trend: (1) a short sub-vertical tangent stage, (2) a knee (i.e., a decrease of curve tangent), and (3) a long sub-horizontal tangent branch (plateau). In the test $A_{l}$, the relative entropy significantly increases after the plateau (i.e., just before the failure). Two main differences can be identified between Shannon entropy and relative entropy graphs: (a) the knees of Shannon entropy curves are smoother than the relative entropy curve ones, and (b) the relative entropy curves reach a very similar value at the failure, which might be assumed as a failure threshold.

(a)
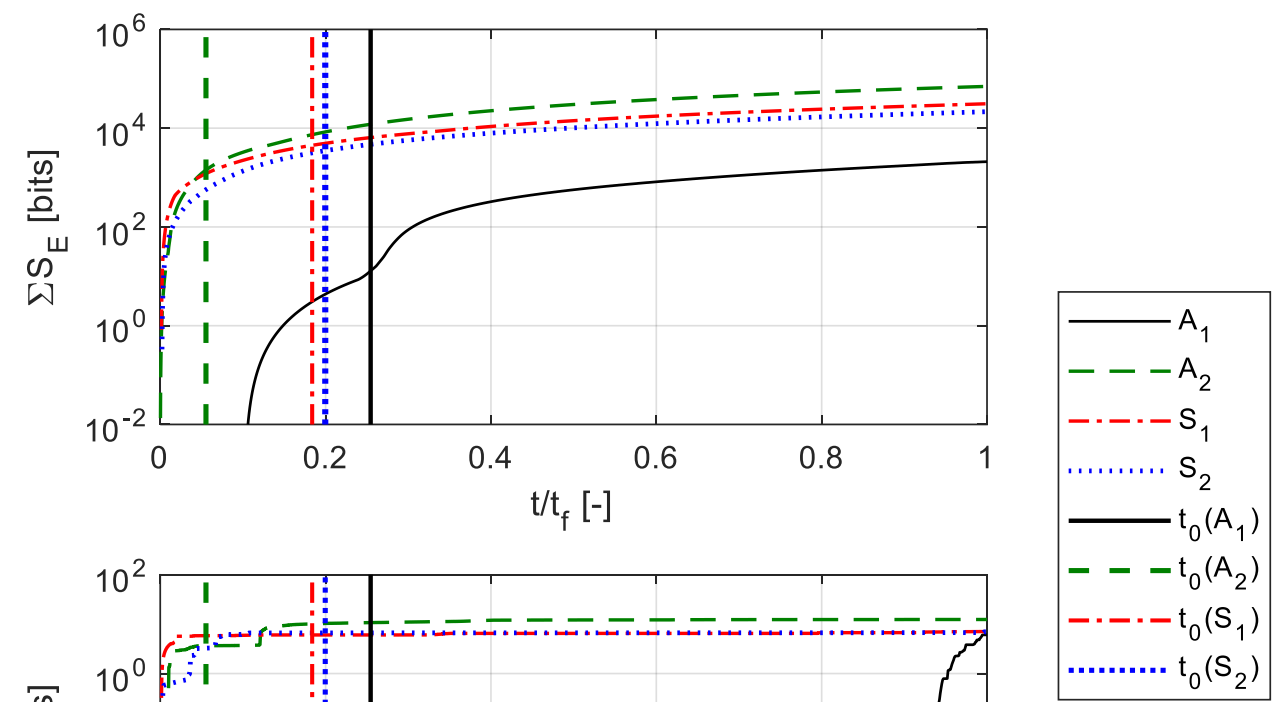

(b)

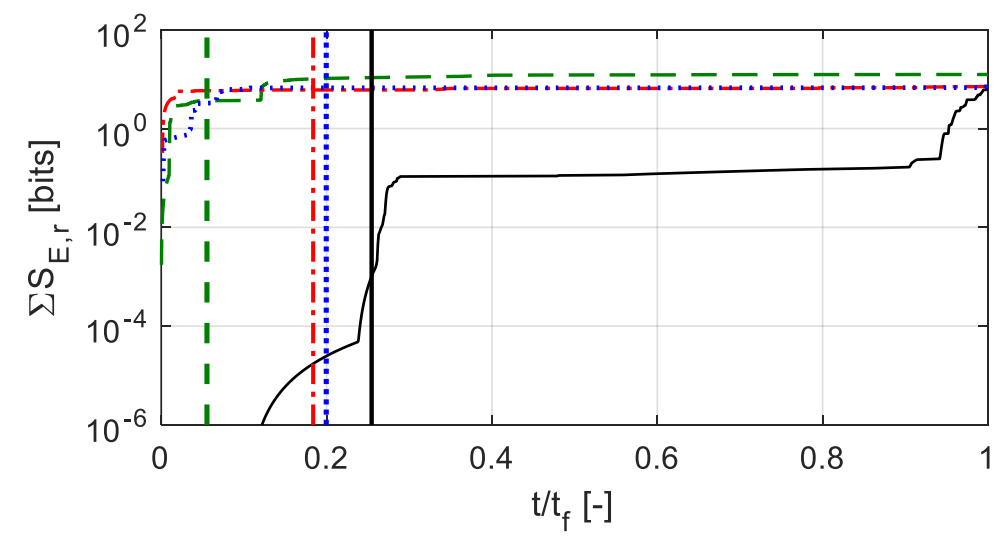

Fig. 5. (a) Cumulative Shannon entropy $\Sigma S_{E}$ and (b) cumulative relative entropy $\Sigma S_{E, r}$.

The crack onset is well correlated to both cumulative entropies. This occurs (just) after the knee as well as significantly before the plateau if Shannon entropy is considered. In the case of the relative entropy, the crack onset is associated with the beginning of the plateau. The failure is identified by considering both entropy formulations: (a) onset of the plateau in the case of Shannon entropy and (b) threshold value in the case of the relative entropy. The failure correlation related to Shannon entropy curves can be considered to be more reliable and consistent than the relative entropy one. In the latter case, the failure threshold value assumes very similar values along the plateau by causing false alarms. A threshold value seems to be a 
necessary but not sufficient condition for the failure. The failure condition observed with regard to Shannon entropy curves is more gradual and stable, i.e., the curve tangent smoothly decreases along with the fracture propagation process (e.g., it can be considered to be a damage index). Moreover, the pattern does not depend on the sample/testing conditions, as well as this is not affected by the noise and the chaotic nature of the acoustic phenomena.

Similar results were recently found by the authors with regard to tensile tests of ductile cast irons ${ }^{43,55}$. In particular, very similar entropy trends over the damage evolution were identified by considering both Shannon and Kullback-Leibler (relative) formulations, despite the significantly different tested material and loading conditions. This strengthens the robustness of the assessment approach.

\subsection{Periodic acoustic entropy}

A simple procedure was defined in MATLAB ${ }^{56}$ to generate unbiased random periodic (or timediscontinuous) monitoring processes. Each process consisted of a number of consecutive discrete time-intervals in which the AE activity was assumed to be detected; such time-intervals were defined as detection windows. No AE signals were assumed to be detected outside the detection windows. The number of detection windows over the whole monitoring process $\left(N_{D W}\right)$ and the duration of each detection window $\left(T_{D W}\right)$ are the main features that define the entire (random-based) monitoring process. $T_{D W}$ is assumed to be constant all over the monitoring process; this has a quite small value if compared to the structural lifetime of the components $\left(L_{T}\right)$. The minimum time distance between the end of the $i^{\text {th }}$ detection window and the beginning of the $(i+1)^{\text {th }}$ detection window is equal to $10 T_{D W}$. A large number of random periodic monitoring processes was generated according to the defined rules/features through MATLAB. Equations 2 and 4 are applied over each detection window along with the defined random periodic monitoring processes. The procedure was repeated a large number of times (i.e., each time considering a different random process) for all performed fatigue fracture tests to simulate unbiased random health monitoring of structures (the detection windows represent the (random-based) periodic inspections during which AE testing is applied).

Fig. 6 shows representative results of (time-)discontinuous Shannon entropy evaluation over the random periodic monitoring processes previously defined. In particular, cumulative Shannon entropy was evaluated for 200 processes considering the test $A_{2}$. The influence of $N_{D W}$ and $T_{D W}$ on the entropy evaluation was assessed. In Fig. 6.a (7.b), red, blue and green plots show the cumulative entropy curves (evaluated for all the random monitoring processes) 
related to $T_{D W}\left(N_{D W}\right)$ equal to 10,20 , and $30 \mathrm{~s}(15,20$, and 25$)$, respectively. The related total AE detection times $T_{T}$ over the lifetime $L_{T}$ were equal to $2.9,5.7$, and $8.6 \%(4.3,5.7$, and 7.1 $\%$ ), respectively. The curves have the same trend of the (time-)continuous cumulative entropy curve (dashed line). The overall data dispersion is not significantly affected by $N_{D W}$ and $T_{D W}$. The entropy values (e.g., the plateau of the curves) increase with $T_{T}$. The different curves tend to reduce their scattering along with the time even though the random process monitoring curves ignore more than $90 \%$ of the recorded $\mathrm{AE}$ data. The time location of the detection windows does not affect the overall curve. The curve knee (i.e., crack onset descriptor) can be observed in all cases; moreover, all curves of the same set achieve approximately constant value before the failure (i.e., identifying the onset of the plateau). Similar results were found for the other considered cases. The results confirm that this approach can be reliable even though (a) (time-)discontinuous AE data monitoring is considered, (b) the detection windows are randomly (time-)spaced, and (c) the total detection time data is smaller than $10 \%$ of the lifetime.

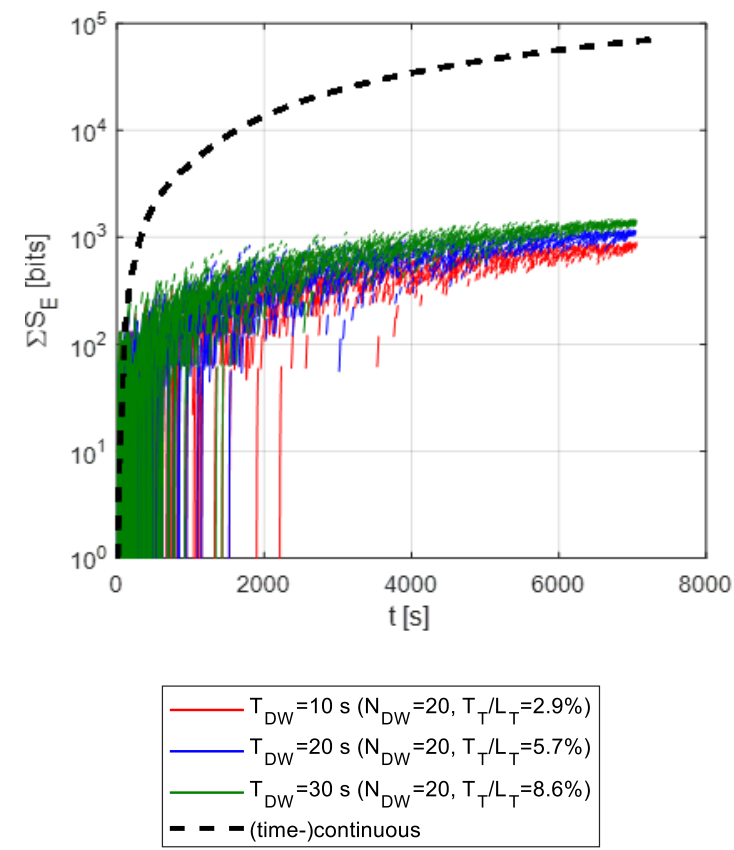

(a)

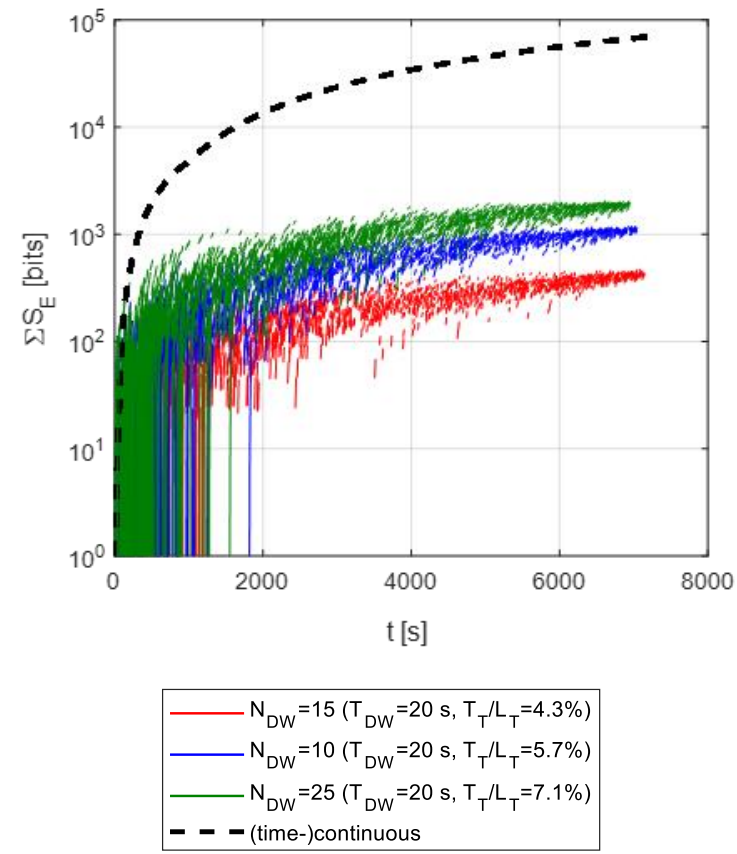

(b)

Fig. 6. Random (time-)discontinuous (or periodic) cumulative Shannon entropy $\left(\Sigma S_{E}\right)$ related to test $A_{2}$ considering different (a) number of detection windows ( $\mathrm{N}_{\mathrm{DW}}$ ) and (b) duration of detection windows $\left(T_{D W}\right)$. Each series of plot (e.g., $T_{D W}=10 \mathrm{~s}\left(N_{D W}=20, T_{T} / L_{T}=2.9 \%\right)$ ) includes 200 random process curves. The (time-)continuous entropy curves are also shown. 


\section{STRUCTURAL HEALTH MONITORING PROTOCOL}

This section presents the application of the above-described methodology in the framework of structural health monitoring. In particular, an innovative monitoring protocol is defined by assuming that the performed FCG tests are representative of fracture-critical components of structures subjected to fatigue loading. As a matter of fact, the main aspects of fatigue crack propagation phenomena are not typically affected by the size, and FCG tests on CT specimens are typically performed to assess the fatigue performance of various materials and structures under fatigue loading. ${ }^{57-59}$

The protocol builds on the (time-)discontinuous evaluation of the cumulative Shannon AE entropy. The protocol monitoring process is defined by sequential detection windows, analogously to the random periodic monitoring processes previously defined. The features of the protocol monitoring process are more realistic than the random process ones for two reasons: (a) the monitoring durations and times are compatible with typical regulation/practice prescriptions and (b) the criteria specifications depend on the real-time entropy evaluation (e.g., health state results depending). The features and the criteria of the protocol are based on the experimental finding as well as on common practice and field research ${ }^{29,60}$

The protocol is based on the (real-time) evaluation of the cumulative entropy $\left(\Sigma S_{E}\right)$ over the detection windows. The variation of the cumulative entropy over the whole $i^{\text {th }}$ detection window $\left(\triangle \Sigma S_{E, i}\right)$ is compared to the previous values in order to define two main damage state: the crack onset and the failure. The damage states are identified by means of multiple conditional tests, i.e., crack onset test:

$\Delta \Sigma \mathrm{S}_{\mathrm{E}, \mathrm{i}} \leq \mathrm{k}_{\Delta 0} \Delta \Sigma \mathrm{S}_{\mathrm{E}, \mathrm{i}-1}$

and failure test:

$\Delta \Sigma \mathrm{S}_{E, i} \leq \mathrm{k}_{\Delta \mathrm{F}} \Delta \Sigma \mathrm{S}_{\mathrm{E}, 345}$

The crack onset test has positive result (corresponding to the $i^{\text {th }}$ detection window) if the cumulative entropy variation over the whole $i^{\text {th }}$ detection window $\left(\Delta \Sigma S_{E, i}\right)$ is smaller than or equal to a threshold value, which is defined as a fraction of the value related to the previous detection window $\left(\Delta \Sigma S_{E, i-1}\right)$ by applying the crack onset set coefficient $k_{\Delta 0}$. The failure test has a positive result (corresponding to the $i^{\text {th }}$ detection window) if the cumulative entropy variation over the whole $i^{t h}$ detection window $\left(\Delta \Sigma S_{E, i}\right)$ is smaller than or equal to an aliquot of $\Delta \Sigma S_{E, 345}$, obtained by applying the failure test coefficient $k_{\Delta F} . \Delta \Sigma S_{E, 345}$ is defined as the average of the 
values related to the $3^{\text {rd }}, 4^{\text {th }}$, and $5^{\text {th }}$ detection window variations evaluated over the entire monitoring process (i.e., $\operatorname{avg}\left\{\Delta \Sigma S_{E, 3}, \Delta \Sigma S_{E, 4}, \Delta \Sigma S_{E, 5}\right\}$ ). The reference detection windows should be the earliest possible over the monitoring process since they have to interpret the least damage condition of the structure (e.g., beginning of structure functioning, or more generally, undamaged structure). However, the first two windows were not considered as a reference since the monitoring process becomes effective for $i$ larger or equal to three by definition. Furthermore, the entropy variation associated with the very beginning of the activity detection is typically associated with extremely large values since the incipient entropy values are extremely reduced, as it can be seen in Fig. 5.a. However, the use of earlier windows to compute the reference entropy variation of the failure criterion might be considered with due consideration, even though it is discouraged by the authors.

The test results of three consequent detection windows (considering the current $i^{t h}$ response as the last of the three) define the time interval $T$ from the end of the $i^{\text {th }}$ detection window to the beginning of the $(i+1)^{\text {th }}$ one, e.g., the time to wait to perform the next AE monitoring inspection. The test results of three consequent windows also identify the alarms to be triggered with the consequent actions to be undertaken. The criteria for timing and the alarms for both crack onset and failure are shown in Tables 4 and 5, respectively.

Table 4. Main criteria for the definition of inspection times, alarms, and actions to be undertaken related to the crack onset assessment.

\begin{tabular}{|c|c|c|c|c|c|}
\hline \multicolumn{4}{|c|}{$\begin{array}{l}\text { crack onset test } \\
\Delta \Sigma S_{E, i} \leq k_{\Delta 0} \Delta \Sigma S_{E, i-1}\end{array}$} & \multirow[t]{2}{*}{$T$} & \multirow[t]{2}{*}{ alarms (actions to be undertaken) } \\
\hline \multirow[t]{9}{*}{ test response } & $\mathrm{i}-2$ & $\mathrm{i}-1$ & $\mathrm{i}$ & & \\
\hline & 0 & 0 & 0 & $T_{1}$ & - \\
\hline & 0 & 0 & 1 & $T_{2}$ & - \\
\hline & 0 & 1 & 0 & $T_{2}$ & - \\
\hline & 0 & 1 & 1 & $T_{3}$ & potential crack onset alarm \\
\hline & 1 & 0 & 0 & $T_{2}$ & - \\
\hline & 1 & 0 & 1 & $T_{3}$ & - \\
\hline & 1 & 1 & 0 & $T_{3}$ & potential crack onset alarm \\
\hline & 1 & 1 & 1 & $T_{4}$ & definite crack onset alarm (special inspection) \\
\hline
\end{tabular}

The time $T$ will decrease if the index increases (e.g., $T_{2}<T_{1}$ ) because if there is the risk of damage in the structure, the inspections and the following AE testing should occur in a shorter period of time. The test response assumes a value equal to $1(0)$ if the conditional test is positive (negative). The potential crack onset alarm and the definite crack onset alarm are caused by two and three consecutive positive crack onset conditional tests over the three windows 
assessed, respectively. The potential failure alarm and the definite failure alarm are caused by one and two (not necessarily consecutive) positive failure conditional test(s) over the three windows assessed, respectively. A special inspection (i.e., localised visual inspection of the monitored components) is required in case of definite crack onset alarm or potential failure alarm. If significant damage is detected during the special inspections, or in case of definite failure alarm, the service/functioning of the structure (or, more generally, of the monitored component) has to be immediately interrupted. This action would allow preventing local/global structural collapse, as well as it would trigger a more direct/invasive monitoring action focussed on the member of interest.

Table 5. Main criteria for the definition of inspection times, alarms, and actions to be undertaken related to the failure assessment.

\begin{tabular}{|c|c|c|c|c|c|}
\hline \multicolumn{4}{|c|}{$\begin{array}{l}\text { failure test } \\
\Delta \Sigma S_{E, i} \leq k_{\Delta F} \Delta \Sigma S_{E, 345}\end{array}$} & \multirow[t]{2}{*}{$T$} & \multirow[t]{2}{*}{ alarms (actions to be undertaken) } \\
\hline \multirow[t]{4}{*}{ test response } & $\mathrm{i}-2$ & $\mathrm{i}-1$ & $\mathrm{i}$ & & \\
\hline & 0 & 0 & 1 & $T_{5}$ & potential failure alarm (special inspection) \\
\hline & 0 & 1 & 1 & - & definite failure alarm (service interruption) \\
\hline & 1 & 0 & 1 & - & definite failure alarm (service interruption) \\
\hline
\end{tabular}

The flow chart in Fig. 7 shows the main steps of the proposed monitoring protocol implemented. Five scalar and three vectorial indexes are considered. The scalar $i$ indicates the number of the (current) inspection and the data detection window; $j$ and $k$ define the number of occurred potential and definite crack onset alarm, respectively; $l$ and $m$ define the number of occurred potential and definite failure alarm, respectively. The indexes $k, l$ and $m$ can be equal to either 1 or 0 since the related alarms can only occur one time. The vector $\underline{\Delta S}$ contains the entropy variation values evaluated for all the detection windows up to the $i^{\text {th }}$ one. The vector $\underline{T}$ records all the time intervals $(T)$ for all the detection windows up to the $i^{\text {th }}$ one; the values of $\underline{T}$ can be equal to $T_{1}, T_{2}, T_{3}, T_{4}$, and $T_{5}$. The vector $\underline{\Delta}$ contains all the responses of the onset/failure tests at all the detection windows up to the $i^{\text {th }}$ one. In particular, the values of $\underline{\Delta}$ can be equal to 0 (negative test response), 1 (positive response at the onset test), and 2 (positive response at the failure test). Both $\underline{T}$ and $\underline{\Delta}$ vectors are updated after each conditional test. All the scalar indexes are set equal to zero at the beginning of the monitoring process, and the vectorial indexes are defined by null vectors. 


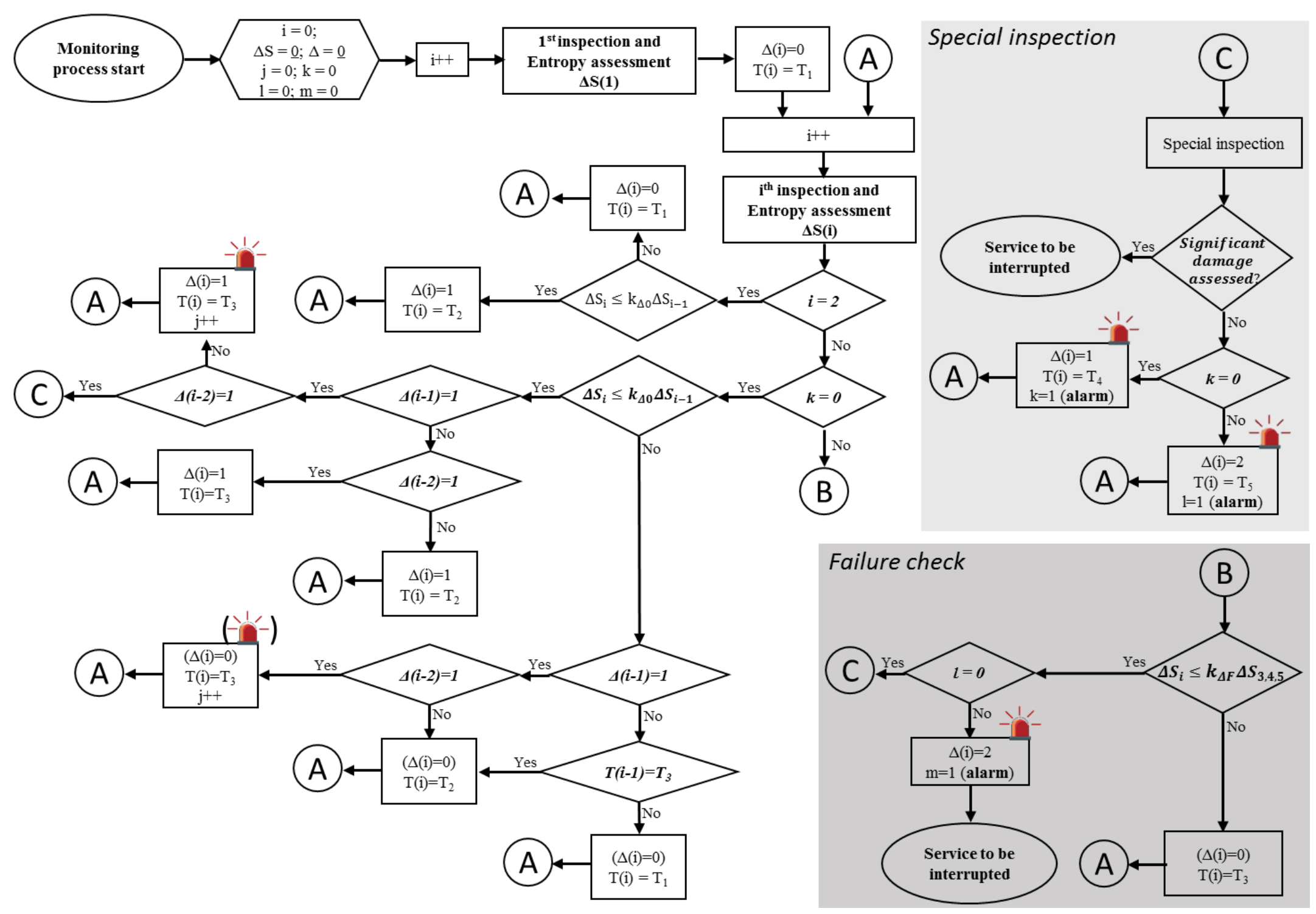

Fig. 7. Flow chart of the developed monitoring protocol. 
The monitoring criteria were applied to the performed fatigue tests. The selected timing parameters are reported in Table 6. Two realistic values for $L_{T}$ were considered: 50 and 100 years. $T_{D W}$ was assumed to be equal to $0.005 L_{T}$. Realistic values $T_{1}$ to $T_{5}$ were chosen as a fraction of $L_{T}$. A reasonable ordinary inspection time interval (e.g., $T_{l}$ ) ranges between two and five years. ${ }^{60}$ Therefore, the assumed values of $T_{l}$ can be considered on the safe side. However, the time parameters can be selected according to the required safety condition for the structure, and in compliance with local guidelines. As an example, a decrease of $T_{l}$ is likely to determine an earlier detection of fracture onset. Similarly, a decrease of $T_{5}$ would give more importance to the potential failure alarm. Both actions would certainly increase the safety/reliability of the monitoring process.

Table 6. Selected set of timing parameters.

\begin{tabular}{lllllll}
\hline$L_{T}$ & $\boldsymbol{T}_{\boldsymbol{D W}}$ & $\boldsymbol{T}_{\boldsymbol{1}}$ & $\boldsymbol{T}_{\mathbf{2}}$ & $\boldsymbol{T}_{\boldsymbol{3}}$ & $\boldsymbol{T}_{\boldsymbol{4}}$ & $\boldsymbol{T}_{\boldsymbol{5}}$ \\
\hline [years] & $\mathbf{0 . 0 0 5} \boldsymbol{L}_{\boldsymbol{T}}$ & $\mathbf{0 . 1} \boldsymbol{L}_{\boldsymbol{T}}$ & $\mathbf{0 . 5} \boldsymbol{T}_{\boldsymbol{1}}$ & $\mathbf{0 . 5} \boldsymbol{T}_{\mathbf{2}}$ & $\mathbf{0 . 5} \boldsymbol{T}_{\mathbf{3}}$ & $\mathbf{0 . 5} \boldsymbol{T}_{\boldsymbol{4}}$ \\
\cline { 2 - 7 } & [months] & [years] & [years] & [months] & [months] & [days] \\
\hline 50 & 3 & 5 & 2.5 & 15 & 7.5 & 112.5 \\
\hline 100 & 6 & 10 & 5 & 30 & 15 & 225 \\
\hline
\end{tabular}

The optimal values for the coefficients $k_{\Delta 0}$ and $k_{\Delta F}$ depend on (a) the typology and material of the component (e.g., metallic plate), (b) the type of damage (e.g., fatigue fracture), and (c) the level of target safety (e.g., time advance for failure prediction). $k_{\Delta 0}$ and $k_{\Delta F}$ were assumed equal to 0.80 and 0.15 as a result of an extensive calibration study on the performed experimental results. The level of safety (e.g., the ratio between the actual failure time and the failure alarm time) is the main variable for the selection of such parameters. In this case, the upper bound limit of the values giving the smallest delay of the crack onset alarm detection was chosen for $k_{\Delta 0}$, i.e., smaller values of $k_{\Delta 0}$ did not reduce (further) the alarm delay. The influence of $k_{\Delta F}$ was particularly significant on the quantitative earliness of the identification of the failure. The failure alarm time, i.e., time in which the failure alarm occurs $\left(\Sigma T_{F}\right)$, divided by the lifetime $(L T)$, was assumed as a calibration parameter for the selection of $k_{\Delta F}$.

Fig. 8 shows $\Sigma T_{F} / L_{T}$ as a function of $k_{\Delta F}$ for all considered cases. The trend of $\Sigma T_{F} / L_{T}$ over $k_{\Delta F}$ was quite similar for the different cases, showing an approximately linear (decreasing) trend. This stable and regular response strengthens the robustness of the protocol. The assumed value for $k_{\Delta F}(0.15)$ corresponds to $\Sigma T_{F} / L_{T}$ ranging between 0.54 and 0.66 , with a mean value equal to 0.62 , e.g., failure triggered in average 19 (38) years earlier than the experimental failure, if $L_{T}$ equal to 50 (100) years is considered. This early failure prediction does not 
represent an excessively safe condition, especially in case of critical damage mechanisms such as fatigue fracture in metals. The failure alarm is preferable to be triggered in the last part of the stable crack propagation rather than at the onset of the unstable response, therefore sufficiently earlier than the actual failure. In this specific case, the assumed value for $k_{\Delta F}$ allowed to detect failure alarm (just) prior to the high and unstable crack growth rates (e.g., larger than $5 \times 10^{-4}$ to $1 \times 10^{-3} \mathrm{~mm}$ for the tested components/materials) leading to the component failure. Assuming values smaller (larger) than 0.15 would predict the failure with an excessively reduced (large) advance, which might be risky (expensive).

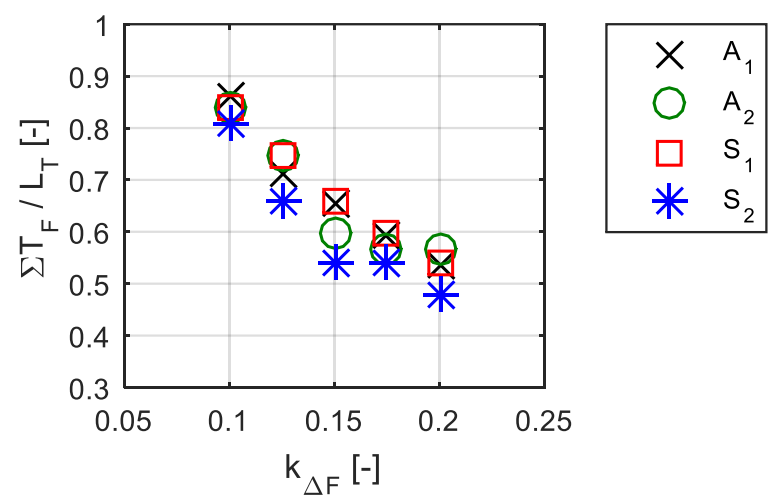

Fig. 8. Failure alarm time $\left(\Sigma T_{F}\right)$ over the lifetime $\left(L_{T}\right)$ as a function of $k_{\Delta F}$ for all cases.

Table 7 reports the results of the monitoring process applied to the case $A_{l}$, where $\underline{\Sigma T}$ is the vector of the total time at the beginning of the inspection. The potential crack onset alarm $(j=$ 1) occurred at the $4^{\text {th }}$ detection window ( $\Sigma T=12.5$ years). The crack onset alarm $(k=1)$ occurred at the $5^{\text {th }}$ detection window ( $\Sigma T=13.8$ years). This triggered the requirement of the special inspection. Assumed that no significant damage was found by the special inspection, the monitoring process proceeded by checking the conditional failure test. Potential failure alarm $(l=1)$ was reached at the $17^{\text {th }}$ detection window ( $\Sigma T=28.8$ years); as a consequence, the special inspection was triggered (the same assumption of no significant damage detected is made). The definite failure alarm $(m=1)$ occurred at the $18^{\text {th }}$ detection window $(\Sigma T=29.2$ years), with the consequent interruption of the structure service.

The application of the monitoring protocol is shown in Fig. 9 for all performed tests, considering the time normalised over the actual failure fatigue time. The cumulative entropy (solid line) is shown as well as the crack onset (triangular markers) and failure alarm occurring (star markers). The (time-)discontinuous entropy (dotted line) and the actual crack onset (vertical dashed line) are also shown, even though they are not considered for the monitoring process but only aimed at post-analysis comparison purposes. The reduced AE activity related 
to the first detection windows determined a small value of the entropy. If no AE activity is detected, the entropy cannot be evaluated (Equation 2). This condition occurred in three cases out of four (i.e., Fig. 9.a, Fig. 9.c, and Fig. 9.d); therefore, the monitoring process did not include the first detection window for all cases. The detected onset alarms (triangular markers) defined the crack onset just after the actual experimental occurrence (dashed vertical line). The late detection of the crack onset observed in Fig. 9.b was caused by the very early occurrence of the crack onset (i.e. between the first and second inspection). Fig. 9.a proves that if the crack onset occurs after the third detection window, the related alarms match with very good agreement the actual crack occurrence. A delay in detecting the crack onset is observed in Fig. 9.b, in which the actual crack onset occurred between the first and the second detection window. In other cases (Fig. s 9.b and 9.c), the actual crack onset occurred between the second and the third inspection. Therefore, the protocol is more efficient if the actual crack onset occurs at least after the second/third inspection, or equivalently, if the monitoring process begins at a time $3 T_{1}$ earlier than the crack onset.

Table 7. Results of the monitoring protocol applied to $A_{l}$ case ( $L_{T}$ equal to 50 years).

\begin{tabular}{|c|c|c|c|c|c|c|}
\hline $\bar{i}$ & $\underline{\Delta}$ & $\underline{T}$ & & $\underline{\Sigma T}$ & alarms (related index) & $\begin{array}{ll}\text { action to be } \\
\text { undertaken }\end{array}$ \\
\hline$[-]$ & {$[-]$} & {$[-]$} & {$\left[L_{T}\right]([$ years $])$} & [years] & {$[-]$} & {$[-]$} \\
\hline 1 & - & $\mathrm{T}_{1}$ & $0.100(5.00)$ & 0.00 & - & - \\
\hline 2 & 0 & $\mathrm{~T}_{1}$ & $0.100(5.00)$ & 5.0 & - & - \\
\hline 3 & 1 & $\mathrm{~T}_{2}$ & $0.050(1.25)$ & 10.0 & - & - \\
\hline 4 & 1 & $\mathrm{~T}_{3}$ & $0.025(0.63)$ & 12.5 & potential crack onset $(j=1)$ & - \\
\hline 5 & 1 & $\mathrm{~T}_{4}$ & $0.013(1.25)$ & 13.8 & definite crack onset $(k=1)$ & special inspection \\
\hline 6 & 0 & $\mathrm{~T}_{3}$ & $0.025(0.63)$ & 14.4 & - & - \\
\hline 7 & 0 & $\mathrm{~T}_{3}$ & $0.025(0.63)$ & 15.7 & - & - \\
\hline 8 & 0 & $\mathrm{~T}_{3}$ & $0.025(0.63)$ & 17.0 & - & - \\
\hline 9 & 0 & $\mathrm{~T}_{3}$ & $0.025(0.63)$ & 18.3 & - & - \\
\hline 10 & 0 & $\mathrm{~T}_{3}$ & $0.025(0.63)$ & 19.6 & - & - \\
\hline 11 & 0 & $\mathrm{~T}_{3}$ & $0.025(0.63)$ & 20.9 & - & - \\
\hline 12 & 0 & $\mathrm{~T}_{3}$ & $0.025(0.63)$ & 22.2 & - & - \\
\hline 13 & 0 & $\mathrm{~T}_{3}$ & $0.025(0.63)$ & 23.5 & - & - \\
\hline 14 & 0 & $\mathrm{~T}_{3}$ & $0.025(0.63)$ & 24.8 & - & - \\
\hline 15 & 0 & $\mathrm{~T}_{3}$ & $0.025(0.63)$ & 26.1 & - & - \\
\hline 16 & 0 & $\mathrm{~T}_{3}$ & $0.025(0.63)$ & 27.5 & - & - \\
\hline 17 & 2 & $\mathrm{~T}_{5}$ & $0.006(0.31)$ & 28.8 & potential failure $(l=1)$ & special inspection \\
\hline 18 & 2 & - & - & 29.2 & definite failure $(m=1)$ & service interruption \\
\hline
\end{tabular}

The early detection of the failure alarm reflected the specific selection of $k_{\Delta F}$, aimed to advance (a) the onset of the unstable crack propagations stage, and (b) excessively large growth rates 
(e.g., larger than $5 \times 10^{-4}$ to $1 \times 10^{-3} \mathrm{~mm}$ ). However, different selections can be made with due considerations, e.g., assuming different values according to Fig. 8, or performing further calibration studies.

(a)

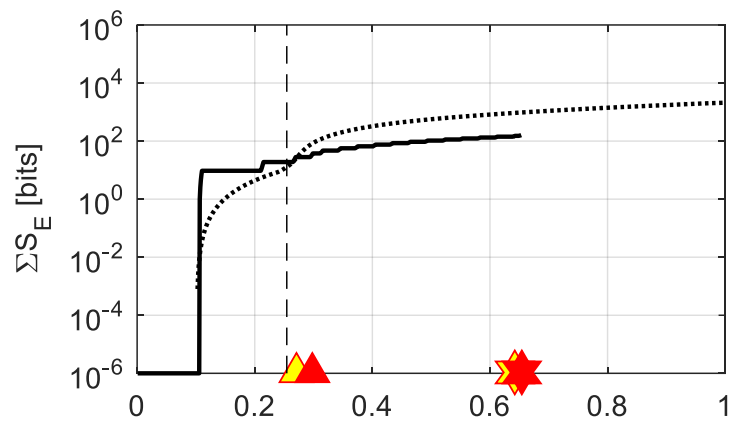

(b)

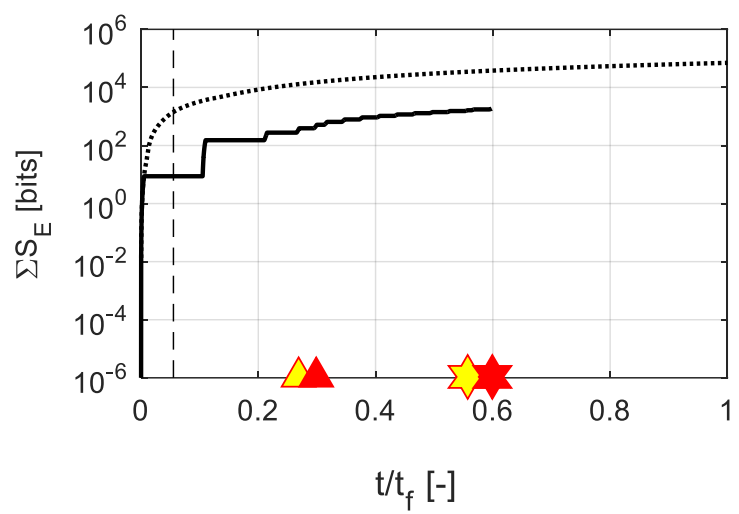

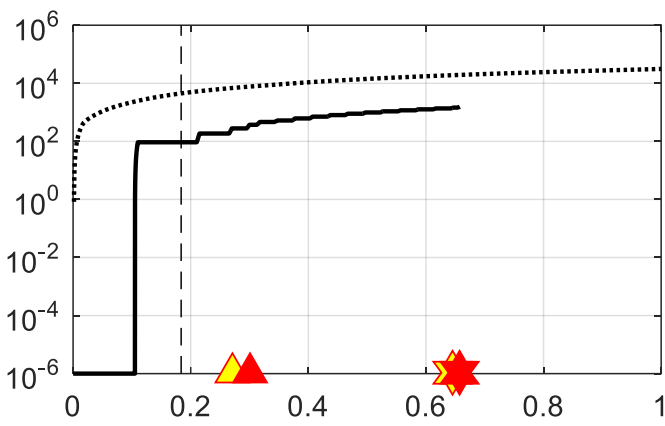

(c)

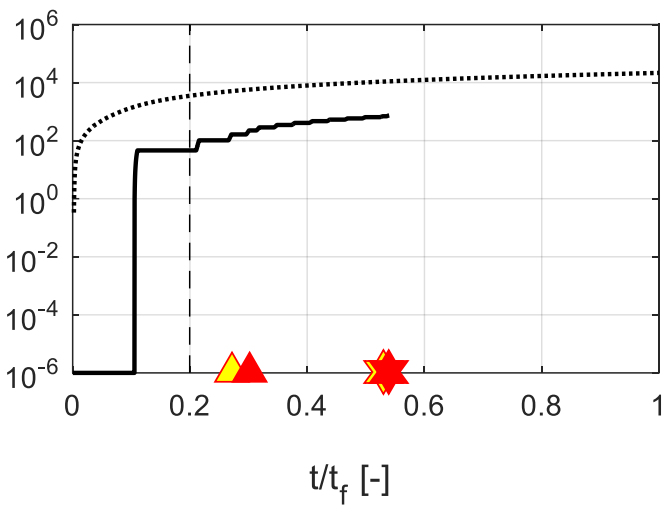

(d)

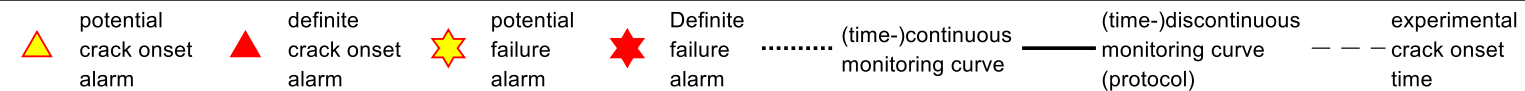

Fig. 9. Application of the proposed monitoring protocol considering the fatigue tests performed: (a) $A_{1}$, (b) $A_{2}$, (c) $S_{1}$, and (d) $S_{2}$.

\section{SUMMARY AND CONCLUSIONS}

An innovative approach was proposed for structural health monitoring of fracture-critical metallic components subjected to fatigue damage. AE testing of FCG in metallic CTs was performed. The tests simulated the condition of a testing environment affected by significant cyclic noise disturbance. The information entropy of the acoustic data was evaluated according to both Shannon and Kullback-Leibler (relative) formulations. Both entropies were found to be clearly correlated to the damage initiation/propagation and failure. The same entropy trend was exhibited over different sample/testing conditions. The assessment of the cumulative entropy allowed to detect the crack onset quantitatively and to predict the incipient fracture failure. Crack onset was identified by (a) a gradual decreasing of the cumulative Shannon entropy and (b) a null slope of the cumulative relative entropy. The accelerated fracture leading to the failure 
was correlated to (a) a sub-horizontal slope of the cumulative Shannon entropy and (b) a threshold value of the cumulative relative entropy. The assessment approach was found to be reliable even though (a) the data detection was (time-)discontinuous (or periodic), (b) the total duration of the monitored data was smaller than $10 \%$ of the component lifetime, and (c) the selection of the data to be detected and analysed was random-based.

An innovative monitoring protocol was developed by considering the evaluation of the cumulative Shannon entropy over realistic monitoring processes based on (time-)discontinuous (or periodic) detection windows. The basic features of the protocol (e.g., duration of the monitoring inspections or ordinary time-interval between consecutive inspections) were derived by regulation prescriptions and current practice approaches. The main novelty of the proposed protocol is that the testing features and the damage criteria depend on the current state of health of the monitored structure. In other words, the assessment criteria are adjusted in real-time as the damage evolves.

The protocol was applied over the performed laboratory tests, which were meant to represent the behaviour of fracture-critical metallic components of structures under fatigue loading within a noisy environment. It is recalled that FCG tests on CTs are typically performed to assess the fatigue and fracture performance of structures and materials (e.g., crack propagation of existing flaw cracks and fracture toughness). Both alarm and timing criteria were defined in the light of $t$ experimental findings and current monitoring practice. Alarm criteria were aimed at assessing the health state of the component and trigger the alarms, whereas timing criteria allowed to establish the time for the next monitoring inspection, as a function of the current health state. The threshold values for the verification of the criteria were defined as a function of the ongoing entropy assessment results. The application of the protocol allowed to (a) define a consistent time interval for the following AE testing inspection and (b) trigger (positively) crack onset and failure alarms. The actual (experimental) crack onset was accurately detected in real-time, especially when the monitoring process had begun longer prior to the damage occurring (monitoring beginning when the structure is undeformed). Failure was predicted in time advance of about $40 \%$ of the component lifetime, as a result of the set level of safety (i.e., the assumed protocol features).

The monitoring approach is flexible: the main parameters of the protocol (e.g., $T_{1}, k_{\Delta 0}$, and $k_{\Delta F}$ ) can be varied according to the required/wanted level of safety, e.g., increasing $k_{\Delta F}$ corresponds to an increase of time advance for failure prediction. The definition of the inspection time according to the ongoing data analysis allows reducing the monitoring costs while keeping a high level of structural safety. The protocol application only considered single 
fracture-critical members under monitoring, but it could reasonably be extended to a set of simultaneously monitored components. The developed monitoring approach is promising for field structural health monitoring even though further tests should be performed considering real loading conditions, e.g. more representative of bridge components under fatigue loading.

\section{SOURCE OF FUNDING}

The project was supported by Seedling 2016, REF 2016/2017, and REF 2017/2018 funds (Dr Marianna Ercolino, University of Greenwich).

\section{CONFLICT OF INTEREST}

The authors declare no conflicts of interest.

\section{AUTHOR CONTRIBUTION}

The research study was conceived, designed, and performed by both authors. DD wrote the paper and ME revised it.

\section{REFERENCES}

1 Vagnoli M, Remenyte-Prescott R, Andrews J. Railway bridge structural health monitoring and fault detection: State-of-the-art methods and future challenges. Structural Health Monitoring. 2018;17: 971-1007. https://doi.org/10.1177/1475921717721137.

2 Aygül M, Bokesjö M, Heshmati M, Al-Emrani M. A comparative study of different fatigue failure assessments of welded bridge details. International Journal of Fatigue. 2013;49: 6272. https://doi.org/10.1016/j.ijfatigue.2012.12.010.

3 Wong L, Rathnayaka S, Chiu WK, Kodikara J. Utilising hydraulic transient excitation for fatigue crack monitoring of a cast iron pipeline using optical distributed sensing. Struct Control Health Monit. 2018;25: e2141. https://doi.org/10.1002/stc.2141.

4 Rafiq MI, Chryssanthopoulos MK, Sathananthan S. Bridge condition modelling and prediction using dynamic Bayesian belief networks. Structure and Infrastructure Engineering. 2014;11: 38-50. https://doi.org/10.1080/15732479.2013.879319.

5 Kosnik DE, Hopwood T, Corr DJ, Thompson DO, Chimenti DE. ACOUSTIC EMISSION MONITORING FOR ASSESSMENT OF STEEL BRIDGE DETAILS. In: San Diego, California, (USA); 2011:1410-1417. https://doi.org/10.1063/1.3592097.

6 Feng Q, Li R, Nie B, Liu S, Zhao L, Zhang H. Literature Review: Theory and Application of In-Line Inspection Technologies for Oil and Gas Pipeline Girth Weld Defection. Sensors. 2016;17: 50. https://doi.org/10.3390/s17010050. 
7 Lee GC, Mohan SB, Huang C, Fard BN. Technical Report MCEER-13-0008 A Study of U.S. Bridge Failures (1980-2012).2013.

8 Wardhana K, Hadipriono FC. Analysis of Recent Bridge Failures in the United States. Journal of Performance of Constructed Facilities. 2003;17: 144-150. https://doi.org/10.1061/(ASCE)0887-3828(2003)17:3(144).

9 Imam B, Chryssanthopoulos MK. A review of metallic bridge failure statistics. In: IABMAS. Philadelphia, USA.; 2010.

10 Yao Y, Tung S-TE, Glisic B. Crack detection and characterization techniques-An overview. Struct Control Health Monit. 2014;21: 1387-1413. https://doi.org/10.1002/stc.1655.

11 Carpinteri A, Vantadori S, Łagoda T, Karolczuk A, Kurek M, Ronchei C. Fatigue assessment of metallic components under uniaxial and multiaxial variable amplitude loading. Fatigue Fract Eng Mater Struct. 2018;41: 1306-1317.

12 Balageas D, Fritzen C-P, Güemes A, eds. Structural Health Monitoring. London; Newport Beach, CA: ISTE; 2006.

13Chen Z, Zhou X, Wang X, Dong L, Qian Y. Deployment of a Smart Structural Health Monitoring System for Long-Span Arch Bridges: A Review and a Case Study. Sensors. 2017;17: 2151. https://doi.org/10.3390/s17092151.

14 Whelan MJ, Gangone MV, Janoyan KD, Jha R. Real-time wireless vibration monitoring for operational modal analysis of an integral abutment highway bridge. Engineering Structures. 2009;31: 2224-2235. https://doi.org/10.1016/j.engstruct.2009.03.022.

15 An Y, Chatzi E, Sim S, Laflamme S, Blachowski B, Ou J. Recent progress and future trends on damage identification methods for bridge structures. Struct Control Health Monit. 2019;26. https://doi.org/10.1002/stc.2416.

16Wisner B, Mazur K, Kontsos A. The use of nondestructive evaluation methods in fatigue: A review. Fatigue Fract Eng Mater Struct. 2020;43: 859-878. https://doi.org/10.1111/ffe.13208.

17 Unnthorsson R. Hit Detection and Determination in AE Bursts. In: Sikorski W, ed. Acoustic Emission - Research and Applications. InTech; 2013. https://doi.org/10.5772/54754.

18 Grosse C, Ohtsu M, eds. Acoustic Emission Testing. Berlin: Springer Berlin Heidelberg; 2008.

19Maslouhi A. Fatigue crack growth monitoring in aluminum using acoustic emission and acousto-ultrasonic methods. Struct Control Health Monit. 2011;18: 790-806. https://doi.org/10.1002/stc.478. 
20 Sauerbrunn C, Kahirdeh A, Yun H, Modarres M. Damage Assessment Using Information Entropy of Individual Acoustic Emission Waveforms during Cyclic Fatigue Loading. Applied Sciences. 2017;7: 562. https://doi.org/10.3390/app7060562.

21 Carpinteri A, Lacidogna G, Niccolini G. Damage analysis of reinforced concrete buildings by the acoustic emission technique. Struct Control Health Monit. 2011;18: 660-673. https://doi.org/10.1002/stc.393.

22 Carpinteri A, Lacidogna G, Manuello A, Niccolini G. A study on the structural stability of the Asinelli Tower in Bologna. Struct Control Health Monit. 2016;23: 659-667. https://doi.org/10.1002/stc.1804.

23Ercolino M, Farhidzadeh A, Salamone S, Magliulo G. Detection of onset of failure in prestressed strands by cluster analysis of acoustic emissions. Structural Monitoring and Maintenance. 2015;2: 339-355. https://doi.org/10.12989/smm.2015.2.4.339.

24Shigeishi M, Colombo S, Broughton KJ, Rutledge H, Batchelor AJ, Forde MC. Acoustic emission to assess and monitor the integrity of bridges. Construction and Building Materials. 2001;15: 35-49. https://doi.org/10.1016/S0950-0618(00)00068-4.

25 Pascoe JA, Zarouchas DS, Alderliesten RC, Benedictus R. Using acoustic emission to understand fatigue crack growth within a single load cycle. Engineering Fracture Mechanics. 2018;194: 281-300. https://doi.org/10.1016/j.engfracmech.2018.03.012.

26Wisner BJ, Potstada P, Perumal VI, Baxevanakis KP, Sause MGR, Kontsos A. Progressive failure monitoring and analysis in aluminium by in situ nondestructive evaluation. Fatigue Fract Eng Mater Struct. 2019;42: 2133-2145. https://doi.org/10.1111/ffe.13088.

27 Nair A, Cai CS. Acoustic emission monitoring of bridges: Review and case studies. $\begin{array}{lll}\text { Engineering } \quad \text { Structures. } & \text { 2010;32: }\end{array}$ https://doi.org/10.1016/j.engstruct.2010.02.020.

28 Nasrollahi A, Deng W, Ma Z, Rizzo P. Multimodal structural health monitoring based on active and passive sensing. Structural Health Monitoring. 2018;17: 395-409. https://doi.org/10.1177/1475921717699375.

29Schultz AE. Protocols and Criteria for Acoustic Emission Monitoring of Fracture-Critical Steel Bridges. Minnesota Department of Transportation; 2015.

30Rosinberg M-L, Vives E. Metastability, Hysteresis, Avalanches, and Acoustic Emission: Martensitic Transitions in Functional Materials. In: Kakeshita T, Fukuda T, Saxena A, Planes A, eds. Disorder and Strain-Induced Complexity in Functional Materials. Vol 148. Berlin, Heidelberg: Springer Berlin Heidelberg; 2012:249-272. https://doi.org/10.1007/978-3-642-20943-7_13. 
31 Kahirdeh A, Khonsari MM. Energy dissipation in the course of the fatigue degradation: Mathematical derivation and experimental quantification. International Journal of Solids and Structures. 2015;77: 74-85. https://doi.org/10.1016/j.ijsolstr.2015.06.032.

32Chai M, Zhang Z, Duan Q. A new qualitative acoustic emission parameter based on Shannon's entropy for damage monitoring. Mechanical Systems and Signal Processing. 2018;100: 617-629. https://doi.org/10.1016/j.ymssp.2017.08.007.

33 Kahirdeh A, Yun H, Modarres M. Degradation Entropy: An Acoustic Emission Based Approach to Structural Health Assessment. https://doi.org/10.12783/shm2017/14112.

34 Kahirdeh A, Sauerbrunn C, Yun H, Modarres M. A parametric approach to acoustic entropy estimation for assessment of fatigue damage. International Journal of Fatigue. 2017;100: 229-237. https://doi.org/10.1016/j.ijfatigue.2017.03.019.

35 Carpinteri A, Lacidogna G, Puzzi S. From criticality to final collapse: Evolution of the "bvalue" from 1.5 to 1.0. Chaos, Solitons \& Fractals. 2009;41: 843-853. https://doi.org/10.1016/j.chaos.2008.04.010.

36 Mousavi M, Holloway D, Olivier JC, Alavi AH, Gandomi AH. A Shannon entropy approach for structural damage identification based on self-powered sensor data. Engineering Structures. 2019;200: 109619. https://doi.org/10.1016/j.engstruct.2019.109619.

37 Yun H, Modarres M. Measures of Entropy to Characterize Fatigue Damage in Metallic Materials. Entropy. 2019;21: 804. https://doi.org/10.3390/e21080804.

38Shannon CE. A Mathematical Theory of Communication. Bell System Technical Journal. 1948;27: 379-423. https://doi.org/10.1002/j.1538-7305.1948.tb01338.x.

39Unnthorsson R, Runarsson TP, Jonsson MT. AE entropy for the condition monitoring of CFRP subjected to cyclic fatigue. J Acoust Emiss. 2008: 262-70.

40Kahirdeh A, Khonsari M. Acoustic Entropy of the Materials in the Course of Degradation. Entropy. 2016;18: 280. https://doi.org/10.3390/e18080280.

41 Kullback S, Leibler RA. On Information and Sufficiency. The Annals of Mathematical Statistics. 1951;22: 79-86. https://doi.org/10.1214/aoms/1177729694.

42 Karimian SF, Modarres M, Bruck HA. A new method for detecting fatigue crack initiation in aluminum alloy using acoustic emission waveform information entropy. Engineering $\begin{array}{lll}\text { Fracture } & \text { Mechanics. } & 106771 .\end{array}$ https://doi.org/10.1016/j.engfracmech.2019.106771.

43 D’Angela D, Ercolino M, Bellini C, Di Cocco V, Iacoviello F. Characterisation of the damaging micromechanisms in a pearlitic ductile cast iron and damage assessment by 
acoustic emission testing. Fatigue Fract Eng Mater Struct. 2020;43: 1038-1050. https://doi.org/10.1111/ffe.13214.

44D'Angela D, Ercolino M. Acoustic Emission Entropy as a fracture-sensitive feature for realtime assessment of metal plates under fatigue loading. Procedia Structural Integrity. 2019;18: 570-576. https://doi.org/10.1016/j.prostr.2019.08.201.

45 Al-Jumaili SKJ. Damage Assessment In Complex Structures Using Acoustic Emission. 2016.

46European Committee for Standardization. EN 2002-16. Aerospace Series - Metallic Materials; Test Methods - Part 16: Non-Destructive Testing, Penetrant Testing.2000.

$47 \mathrm{Yu}$ J, Ziehl P, Zárate B, Caicedo J. Prediction of fatigue crack growth in steel bridge components using acoustic emission. Journal of Constructional Steel Research. 2011;67: 1254-1260. https://doi.org/10.1016/j.jcsr.2011.03.005.

48Chai M, Zhang J, Zhang Z, Duan Q, Cheng G. Acoustic emission studies for characterization of fatigue crack growth in 316LN stainless steel and welds. Applied Acoustics. 2017;126: 101-113. https://doi.org/10.1016/j.apacoust.2017.05.014.

49Aggelis DG, Kordatos EZ, Matikas TE. Acoustic emission for fatigue damage characterization in metal plates. Mechanics Research Communications. 2011;38: 106-110. https://doi.org/10.1016/j.mechrescom.2011.01.011.

50Colombo IngS, Main IG, Forde MC. Assessing Damage of Reinforced Concrete Beam Using “ b -value” Analysis of Acoustic Emission Signals. Journal of Materials in Civil Engineering. $\quad 2003 ; 15: \quad 280-286 . \quad$ https://doi.org/10.1061/(ASCE)08991561(2003)15:3(280).

51 Gutenberg B, Richter CF. Frequency of earthquakes in California. Bull seism Soc Am. 1944;34: 185-188.

52Rao MVMS, Prasanna Lakshmi KJ. Analysis of b-value and improved b-value of acoustic emissions accompanying rock fracture. Current Science. 2005;89: 1577-1582.

53 Botvina LR, Soldatenkov AP, Tyutin MR, Demina YuA, Levin VP, Petersen TB. On interrelation of damage accumulation in structural steels and physical parameters estimated by methods of acoustic emission and metal magnetic memory. Russian Metallurgy (Metally). 2017;2017: 10-17. https://doi.org/10.1134/S0036029517010037.

54 Stavrakas I, Triantis D, Kourkoulis SK, Pasiou ED, Dakanali I. Acoustic Emission Analysis of Cement Mortar Specimens During Three Point Bending Tests. Latin American Journal of Solids and Structures. 2016;13: 2283-2297. https://doi.org/10.1590/1679-78252486. 
55D'Angela D, Ercolino M, Bellini C, Di Cocco V, Iacoviello F. Assessment of fatigue damage in a fully pearlitic ductile cast iron by evaluation of Acoustic Emission Entropy. $\begin{array}{llll}\text { Procedia Structural } & \text { Integrity. }\end{array}$ https://doi.org/10.1016/j.prostr.2020.04.041.

56 The MathWorks Inc. Matlab 9.5. 2018.

57Farahani BV, Tavares PJ, Belinha J, Moreira PMGP. A Fracture Mechanics Study of a Compact Tension Specimen: Digital Image Correlation, Finite Element and Meshless $\begin{array}{llll}\text { Methods. } & \text { Procedia } & \text { Structural } & \text { Integrity. 2017;5: }\end{array}$ https://doi.org/10.1016/j.prostr.2017.07.113.

58 Roberts TM, Talebzadeh M. Acoustic emission monitoring of fatigue crack propagation. Journal of Constructional Steel Research. 2003;59: 695-712. https://doi.org/10.1016/S0143-974X(02)00064-0.

59Zárate BA, Caicedo JM, Yu J, Ziehl P. Bayesian model updating and prognosis of fatigue $\begin{array}{llll}\text { crack } & \text { growth. } & \text { Engineering } & \text { 53-61 }\end{array}$ https://doi.org/10.1016/j.engstruct.2012.06.012.

60U.S. Department of Transportation (USDOT). National Bridge Inspections Standards Regulation (NBIS).2004. 